
Not for reproduction, distribution or commercial use.

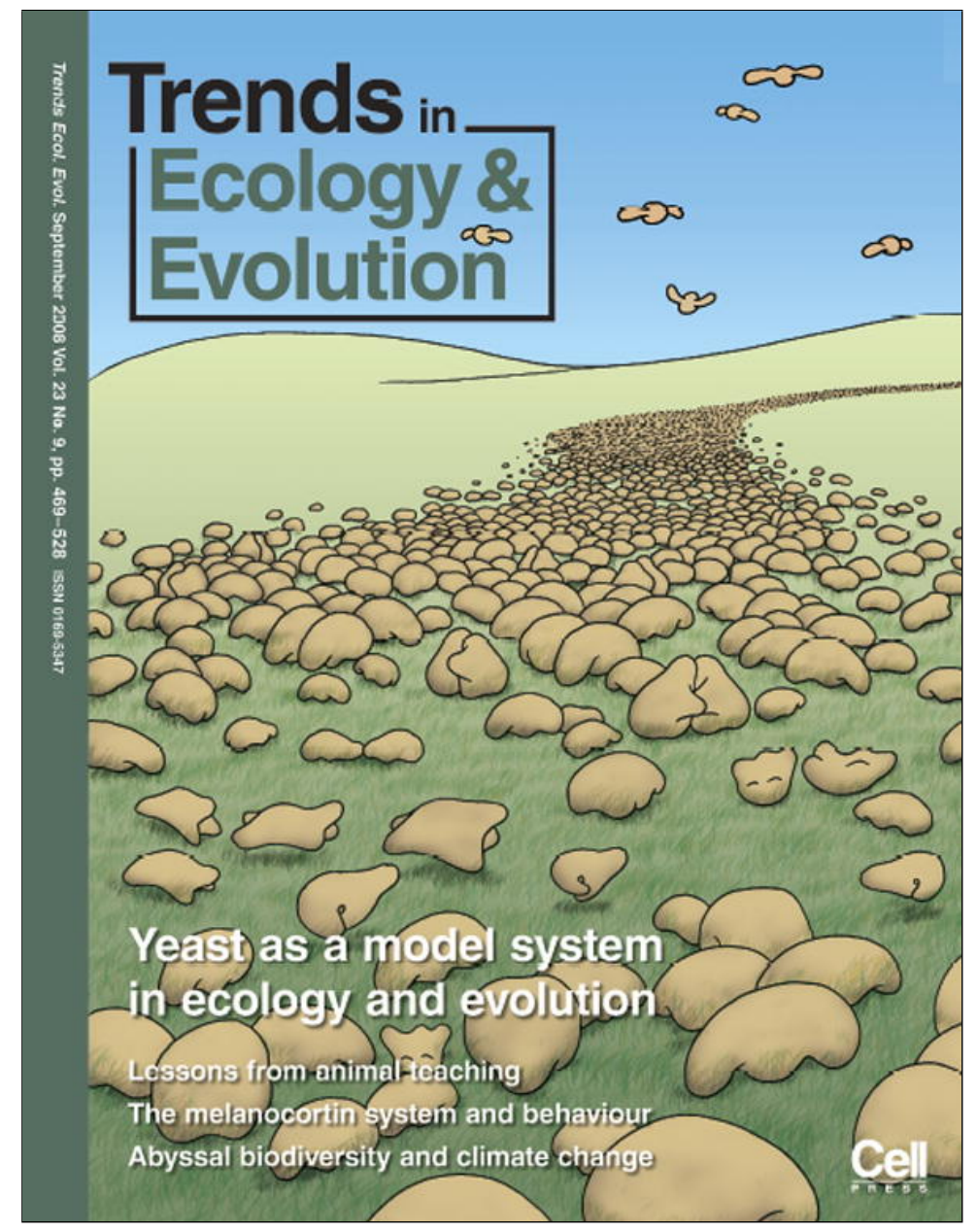

This article appeared in a journal published by Elsevier. The attached copy is furnished to the author for internal non-commercial research and education use, including for instruction at the authors institution and sharing with colleagues.

Other uses, including reproduction and distribution, or selling or licensing copies, or posting to personal, institutional or third party websites are prohibited.

In most cases authors are permitted to post their version of the article (e.g. in Word or Tex form) to their personal website or institutional repository. Authors requiring further information regarding Elsevier's archiving and manuscript policies are encouraged to visit:

http://www.elsevier.com/copyright 


\title{
Pleiotropy in the melanocortin system, coloration and behavioural syndromes
}

\author{
Anne-Lyse Ducrest, Laurent Keller and Alexandre Roulin \\ Department of Ecology and Evolution, University of Lausanne, Biophore, $\mathrm{CH}-1015$ Lausanne, Switzerland
}

\begin{abstract}
In vertebrates, melanin-based coloration is often associated with variation in physiological and behavioural traits. We propose that this association stems from pleiotropic effects of the genes regulating the synthesis of brown to black eumelanin. The most important regulators are the melanocortin 1 receptor and its ligands, the melanocortin agonists and the agouti-signalling protein antagonist. On the basis of the physiological and behavioural functions of the melanocortins, we predict five categories of traits correlated with melanin-based coloration. A review of the literature indeed reveals that, as predicted, darker wild vertebrates are more aggressive, sexually active and resistant to stress than lighter individuals. Pleiotropic effects of the melanocortins might thus account for the widespread covariance between melanin-based coloration and other phenotypic traits in vertebrates.
\end{abstract}

\section{Adaptive function of melanin-based coloration}

Pigmentation of the hair, skin, cuticle, feather and eye, which is mainly determined by brown to black eumelanin and yellow to reddish-brown pheomelanin, is one of the phenotypes that varies most in vertebrates (Figure 1). Melanin-based coloration is frequently implicated in social communication [1] and covaries with many other physiological, morphological and behavioural traits [2]. Whereas some traits such as antibiotic activity and resistance to solar radiation and oxidative stress can directly result from the physico-chemical properties of melanin pigments [3], most of the known associations cannot. For example, darkermaned male lions (Panthera leo) are more aggressive and sexually more active [4], and barn owl (Tyto alba) offspring mount stronger immune responses when their biological parents are darker even when they are raised by foster parents [5], yet the reasons underlying these associations and the fitness consequences remain largely unknown.

Here we propose and provide evidence that pleiotropic effects of key regulators of melanogenesis might be responsible for the widespread association between melaninbased coloration and other phenotypic traits in wild vertebrates. To this end, we first briefly describe the melanocortin system, which primarily consists of the G-protein-coupled melanocortin 1 receptor (MC1R) and its agonists the melanocortins (i.e. melanin-stimulating hormones $\alpha-, \beta$ - and $\gamma$-MSHs and the adrenocorticotropin hormone, ACTH) and its inverse agonist and antagonist the agouti-signalling protein (ASIP). Next, we propose that because melanocortins also bind to four other melanocortin receptors (MC2-5R) which have very different functions (Figures 2,3), the degree of melanin-based coloration should covary with other phenotypic traits. To predict these associations, we review the extensive body of genetic and pharmacological studies on the melanocortin system (Table 1 and Supplementary Table S1). Finally, we examine the literature to determine whether patterns observed in wild populations of vertebrates are consistent with these predictions (Table 2 and Supplementary Table S2) and discuss the evolutionary implications of the widespread pleiotropic effects of the melanocortins.

\section{The melanocortin system}

Melanocortins are posttranslational products of the proopiomelanocortin $(P O M C)$ gene. In vertebrates, the $P O M \bar{C}$ and the five $M C R$ genes are highly conserved and their tissue distribution and functions are similar across species with few exceptions [6]. Successive cell-specific processing and posttranslational modifications of the POMC prohormone generate at least four melanocortin peptides $(\alpha-, \beta-$ and $\gamma$-MSH and ACTH) as well as endorphins [7]. In vertebrates, the principal $M C R$ expressed in the skin and implicated in melanogenesis is $M C 1 R$, but a role for other $M C R$ s cannot be excluded because weak expressions

\section{Glossary}

ACTH: Adrenocorticotropic hormone is a melanocortin which is part of the HPA axis.

Agonist: Molecule that binds to a specific receptor and triggers a response in the cell.

AGRP: Agouti-related protein is an inverse agonist and antagonist at MC3R and MC4R in the brain.

Analogue: Substance that copies one or more action of a molecule. Antagonist: Molecule that prevents the activation of a receptor.

ASIP: Agouti-signalling protein, also referred to as Agouti or Nonagouti in mice, is an inverse agonist and antagonist at MC1R.

Behavioural syndrome: Consistent individual differences in a suite of correlated behaviours.

Inverse agonist: Molecule that exerts the opposite pharmacological effect of a receptor agonist (for example, when MSH binds to MC1R, eumelanin production is induced, whereas when the inverse agonist ASIP binds to MC1R, pheomelanin production is induced).

MCRs: Five melanocortin receptors (MC1-5R) belonging to the rhodopsin family seven-transmembrane, G-protein-coupled receptors.

Melanocortins: Peptidic hormones that include ACTH and MSH derived from the prohormone proopiomelanocortin (POMC). They act through five melanocortin receptors, MC1-5R. Neural and pituitaric melanocortins act as neurocrine and endocrine factors, whereas peripherally produced melanocortins have paracrine and autocrine roles.

MSH: $\alpha$-, $\beta$ - and $\gamma$-melanocyte-stimulating hormones are melanocortins.

Neuroendocrine: Molecule that acts through nerve cells (neurocrine, neurotransmitter) and the vascular system (endocrine, hormone).

Pleiotropy: The phenomenon whereby a single gene affects several apparently unrelated aspects of the phenotype.

Reaction norm: Pattern of phenotypes produced by a given genotype under different environmental conditions. 


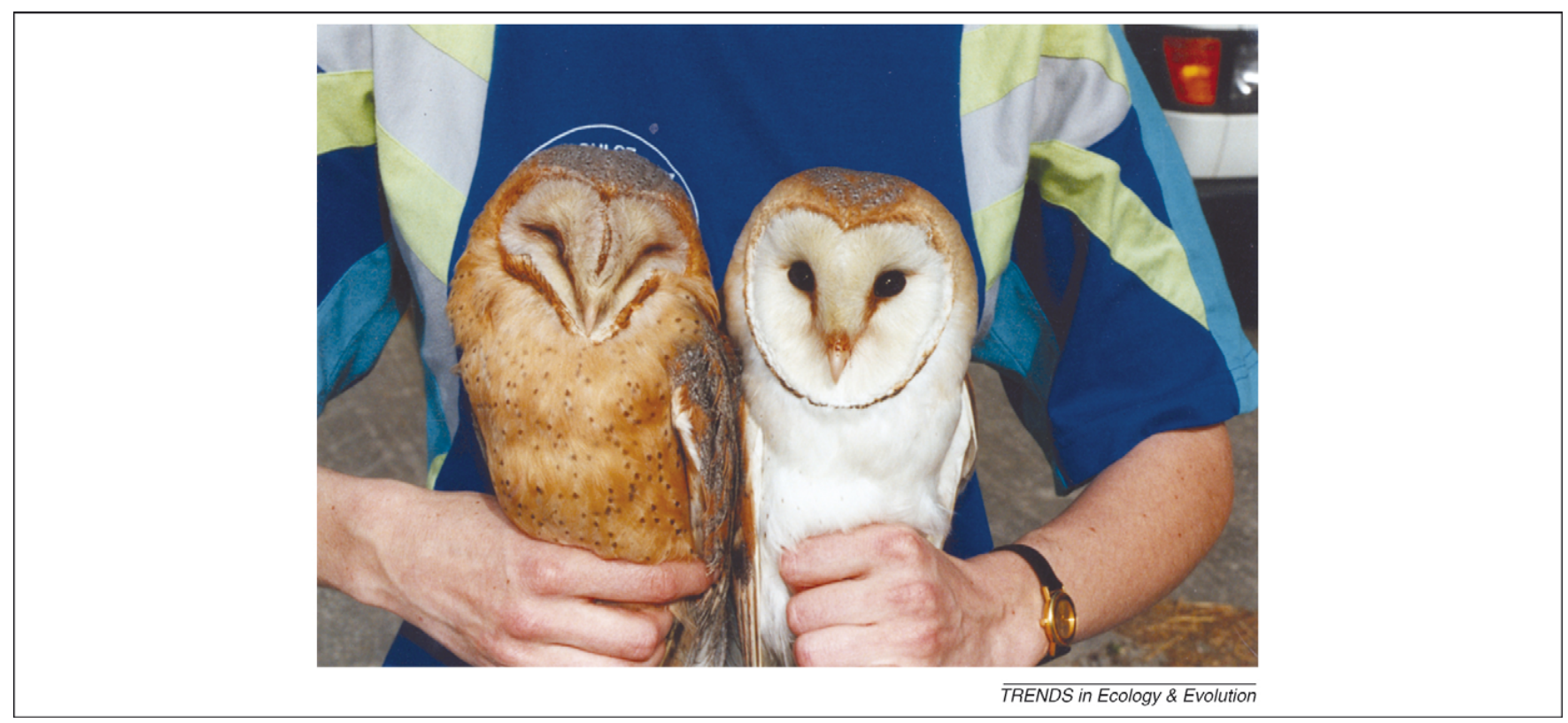

Figure 1. Colour variation in barn owls (Tyto alba). The individual on the left is dark reddish-brown (i.e. pheomelanic) with many large black eumelanic spots, whereas the individual on the right is white (i.e. not pheomelanic) and immaculate.

of the other MCRs are detected in the skin of mammals [8] (Figure 3). The balance between eu- and pheomelanin synthesis is controlled by melanocortin agonists binding to MC1R; by contrast, binding of the natural inverse agonist and antagonist ASIP reduces or blocks eumelanin production and switches on pheomelanin synthesis, result- ing in a yellow band just below the tip of the black or brown hair in wild-type mice [9] (Figure 2). ASIP is also a potent inverse agonist and antagonist at MC4R and a weaker one at MC3R and MC5R [10]. A homologous protein to ASIP called AGRP (agouti-related protein) is a natural inverse agonist and antagonist of melanocortins in the brain [11].

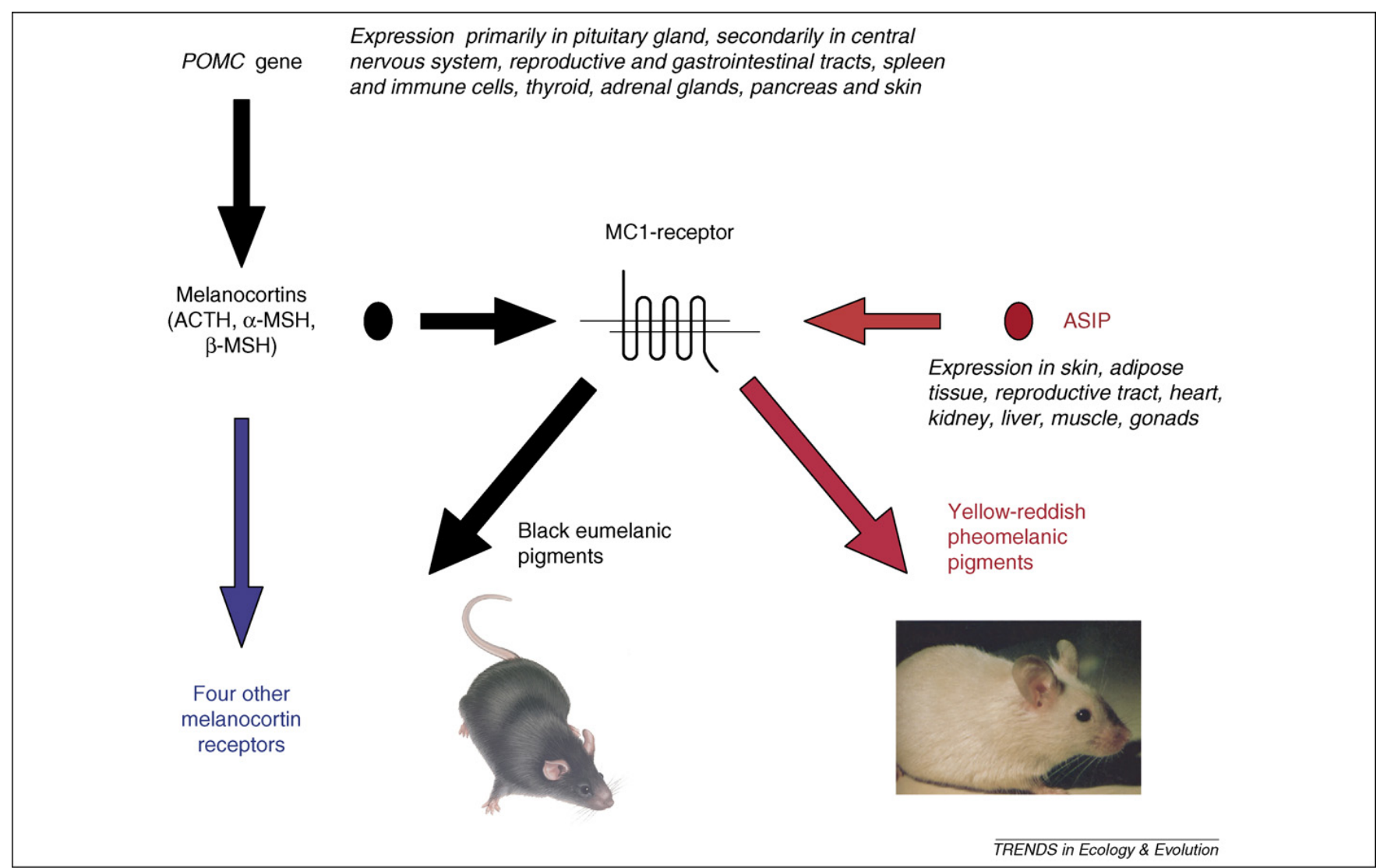

Figure 2. Production of eumelanic and pheomelanic pigments. Binding of the melanocortin agonists to MC1R triggers the production of black eumelanic pigments, whereas binding of the ASIP inverse agonist and antagonist induces the production of yellow-reddish pheomelanic pigments. Melanocortins bind to four other melanocortin receptors (MC2-5R) which have very different functions (Figure 3). Sites where POMC and ASIP are expressed are indicated in italics. 


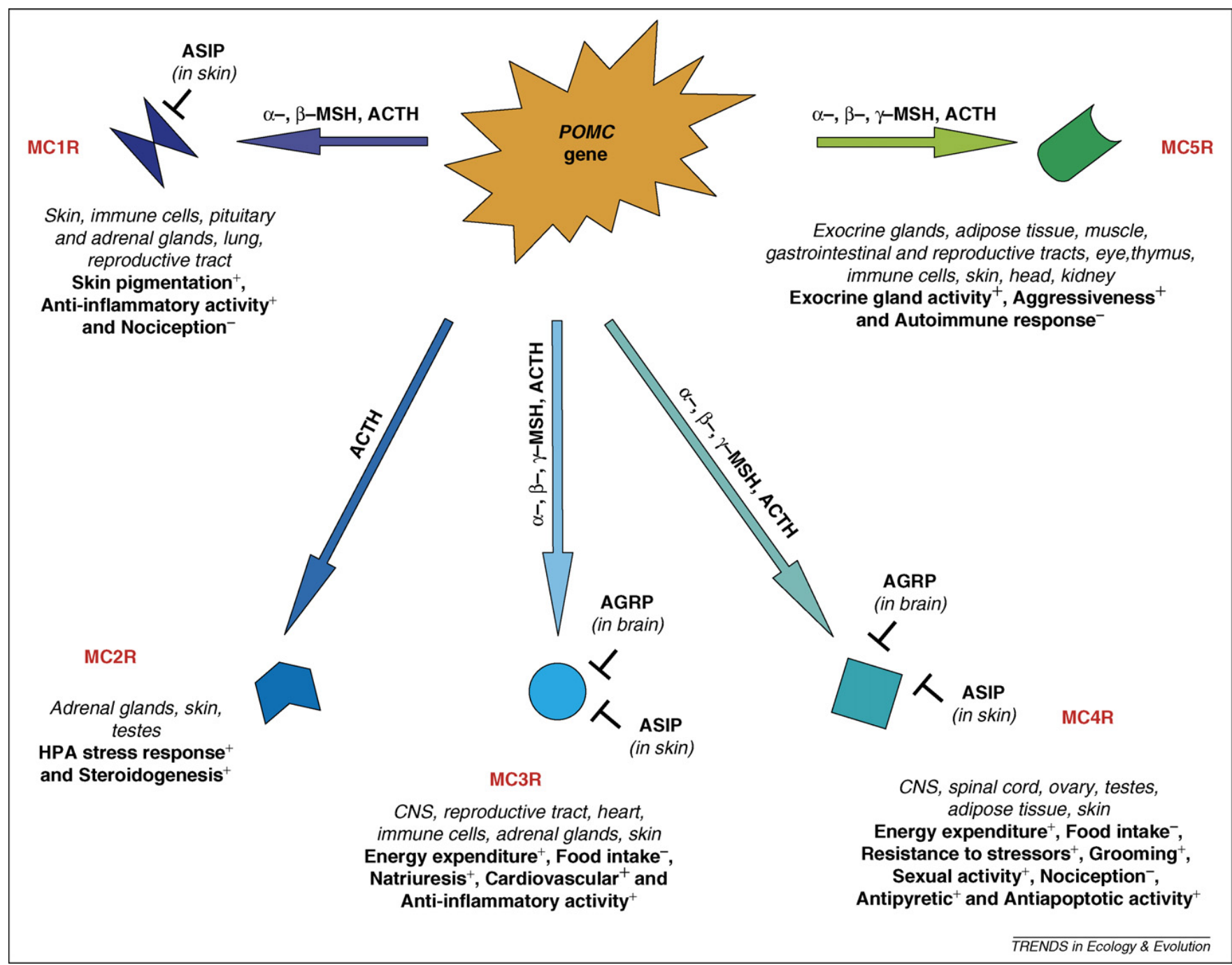

Figure 3. Melanocortin system. The proopiomelanocortin (POMC) gene produces the melanocortins ( $\alpha$-, $\beta$ - and $\gamma$-MSH and ACTH) which bind to five melanocortin receptors (MC1-5R). Location of these five receptors in vertebrates is given in italics while their function is written in bold. For each function, we report whether binding of the melanocortins to the different MCRs has positive $(+)$ or negative effects $(-)$. For example, binding of melanocortins to MC4R increases energy expenditure but reduces food intake. The agonists and inverse antagonists (agouti-signalling protein, ASIP or agouti-related protein, AGRP) for each MCRs are indicated with the symbol $\perp$.

In mammals, AGRP is involved in the control of energy homeostasis and does not affect melanogenesis. AGRP is equally potent to ASIP in binding MC3R and MC4R but has little affinity for MC5R and no affinity for MC1R [10] (Figure 3).

\section{Pleiotropic effects of the melanocortin system}

Because melanocortins bind not only to MC1R but also to MC2-5R which are responsible for several physiological and behavioural functions (Figure 3), this might result in a covariation between melanin-based coloration and other phenotypic traits. To obtain information on the expected covariations, we reviewed murine studies on constitutive and conditional knockouts of POMC (pomc1-/-), MC1R (e/e), MC2-5R (Mcr-/-) and AGRP genes (agrp-/-), as well as dominant agouti mutations (ASIP mutations, $A^{y} / a$ ) and overexpression of ASIP, AGRP and the POMC gene region coding for $\alpha$ - and $\gamma$-MSH (Supplementary Table S1). We also included experiments on the administration of melanocortins, ASIP, AGRP and their analogues in 16 mammals, 3 birds, 1 amphibian and 4 fish species.
An analysis of these studies reveals that many key phenotypic traits are recurrently affected by the manipulation of the genes which are involved in the melanocortin system (or the products of these genes). The phenotypes for which there was a consistent association between the phenotype and the change in activity of the MCRs are listed in Table 1 . On the basis of these associations, we derive predictions in the next sections about how five key categories of physiological and behavioural functions might covary with melanin-based coloration of wild vertebrates (Table 1). For each of the five predictions, we review empirical studies in wild populations of vertebrates reporting covariation between melanin-based coloration and the relevant phenotypic traits (Table 2 and Supplementary Table S2). These studies were conducted in 3 mammals (African lion, soay sheep Ovis aries and white-tailed deer Odocoileus virginianus), 4 fish (mosquitofish Gambusia affinis, guppy Poecilia reticulata, green swordtail Xiphophorus hellerii and Arctic charr Salvelinus alpinus), 4 reptiles (asp viper Vipera aspis, adder V. berus, fence lizard 
Table 1. Summary of the positive and negative pleiotropic effects obtained by manipulations of key components of the melanocortin system and their derived predictions on covariation between eumelanin-based coloration and physiological and behavioural functions in wild vertebrates reported in Supplementary Table S1

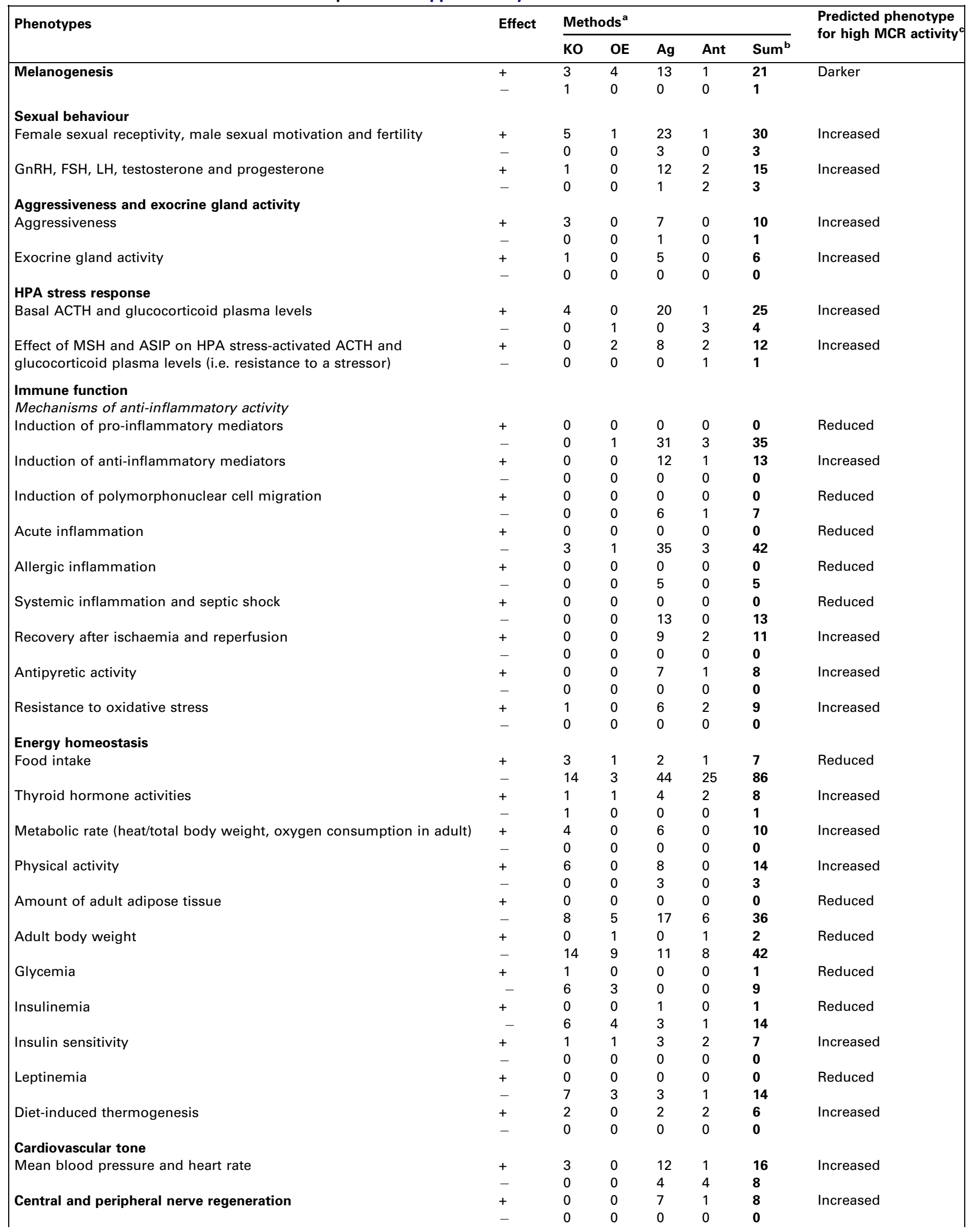


Table 1 (Continued)

\begin{tabular}{|c|c|c|c|c|c|c|c|}
\hline \multirow[t]{2}{*}{ Phenotypes } & \multirow[t]{2}{*}{ Effect } & \multicolumn{5}{|c|}{ Methods ${ }^{a}$} & \multirow{2}{*}{$\begin{array}{l}\text { Predicted phenotype } \\
\text { for high MCR activity }\end{array}$} \\
\hline & & KO & OE & Ag & Ant & Sum $^{b}$ & \\
\hline \multicolumn{8}{|l|}{ Nonsocial behaviour } \\
\hline \multirow[t]{2}{*}{ Grooming } & + & 0 & 0 & 18 & 7 & 25 & Increased \\
\hline & - & 0 & 0 & 1 & 0 & 1 & \\
\hline \multirow[t]{2}{*}{ Stretching and yawning } & + & 0 & 0 & 22 & 1 & 23 & Increased \\
\hline & - & 0 & 0 & 1 & 0 & 1 & \\
\hline
\end{tabular}

GenBank accession numbers for the murine genes are the following: POMC: NM 008895; MC1R: NM 008559; MC2R: NM 008560; MC3R: NM 008561; MC4R: NM 016977; MC5R: NM 013596; ASIP: NM 015770; AGRP: NM 007427.

aPhenotypes showing a positive (+) or negative (-) effect observed for full and conditional knockout mice of POMC, MC1-5R and AGRP genes (KO), for transgenic mice overexpressing POMC, ASIP, AGRP and dominant mutant agouti mice $(\mathrm{OE})$, for animals injected with melanocortins and their analogues (Ag) or with antagonists and their analogues at MCRs (Ant).

${ }^{\mathrm{b}}$ Total number of $\mathrm{KO}, \mathrm{OE}, \mathrm{Ag}$ and Ant studies.

'Predicted phenotypes derived from KO, OE, Ag and Ant studies for high MCR activities in wild vertebrates. For example: increased sexual activity means that a higher level of melanocortin activities increases sexual activity.

Sceloporus occidentalis and spiny lizard S. olivaceus), 1 amphibian (spadefoot toad Spea bombifrons) and 36 bird species.

\section{Melanin-based coloration and sexual behaviour}

Melanocortins enhance fertility, female sexual receptivity and male sexual motivation and performance (Table 1). These effects are mediated partly by ACTH, which increases sexual steroid production through binding to MC2R in adrenal glands, and by all MSHs binding to MC4R through neurocrine pathways including the spinal cord and peripheral nerves [12] (Figure 3). In addition, melanocortins have a positive effect on the production of sexual hormones [13] (Table 1). Based on these effects, we predict that darker eumelanic vertebrates will be sexually more active. In line with these predictions, in all the nine species with available data, darker males were sexually more active than lightercoloured male conspecifics (Table 2). Moreover, in four studies where testosterone level was measured, the level was higher in darker males (Table 2).

\section{Melanin-based coloration, aggressiveness and exocrine gland activity}

Melanocortins promote aggressiveness by inducing the production of aggression self-stimulating pheromones through binding to MC5R [14] (Table 1). Moreover, via MC5R, melanocortins enhance the secretion and excretion of exocrine glands, such as the murine preputial, Harderian, lacrimal and sebaceous glands (Table 1), which, for example, increase the hair lipid content and improve water repulsion of the fur [15]. Accordingly, darker eumelanic individuals should be more aggressive and have an enhanced exocrine gland activity. We found strong support for the prediction on aggressiveness, with dark eumelanic individuals being more aggressive than lighter males for the same population in 18 out of 20 species for which data were available. One study also investigated the relationship between eumelanin-based coloration and the weight of an exocrine gland in the barn owl [16] (Table 2). Contrary to expectation, darker individuals had a lighter uropygial gland, but it remains to be investigated how the weight of the gland correlates with gland content and activity.

\section{Melanin-based coloration and the hypothalamic- pituitary-adrenal stress response}

One of the major regulators of the stress response is the hypothalamic-pituitary-adrenal (HPA) axis which consists of the hypothalamic corticotropin-releasing hormone, which stimulates the pituitaric ACTH and further activates the synthesis of glucocorticoids (cortisol and corti-

Table 2. Summary of the covariation between eumelanin-based coloration and behavioural, physiological and morphological traits in wild vertebrates reported in Supplementary Table S2

\begin{tabular}{|c|c|c|c|}
\hline Category of traits & Phenotypes & $\begin{array}{l}\text { Sign of } \\
\text { covariation }\end{array}$ & $N^{a}$ \\
\hline \multirow[t]{4}{*}{ Sexual traits } & Sexual behaviour & + & 9 \\
\hline & & - & 0 \\
\hline & Plasma testosterone level & + & 4 \\
\hline & & - & 0 \\
\hline \multirow{4}{*}{$\begin{array}{l}\text { Aggressiveness and exocrine } \\
\text { gland activity }\end{array}$} & Aggressiveness & + & 18 \\
\hline & & - & 2 \\
\hline & Mass of uropygial gland & + & 0 \\
\hline & & - & 1 \\
\hline \multirow[t]{2}{*}{ Stress response } & Resistance to stressors & + & 6 \\
\hline & & - & 0 \\
\hline \multirow[t]{2}{*}{ Immune system } & Immune response against nonpathogenic & + & 1 \\
\hline & antigen & - & 0 \\
\hline \multirow[t]{5}{*}{ Energy homeostasis } & Metabolic rate & + & 2 \\
\hline & & - & 0 \\
\hline & Body mass & + & 7 \\
\hline & & - & 3 \\
\hline & & - & 1 \\
\hline
\end{tabular}

${ }^{a}$ Number of studies showing a significant positive or negative correlation between eumelanin-based coloration and other phenotypic attributes. 
costerone) by binding to MC2R in adrenal glands [17]. An equivalent HPA axis also exists in the skin and is responsible for response to local cutaneous stress [18]. As shown in Table 1, stressors induce the HPA stress response, resulting in an increase in circulating ACTH and glucocorticoids. The other melanocortins modulate this general HPA stress response. Through binding to MC4R, $\alpha-\mathrm{MSH}$ increases resistance to stressors whereas ASIP has the opposite effect [19] (Table 1). Therefore, darker eumelanic individuals should be less sensitive to stressful factors. This is the pattern that was found in all of the six species studied (Table 2).

\section{Melanin-based coloration and immune function}

Through binding to MC1R, MC3R and MC5R, melanocortins as well as the $\alpha$-MSH-derived tripeptide $\alpha$-MSH(1113) reduce acute, allergic and systemic inflammation and septic shock, and also improve recovery after ischaemia [20] (Table 1). In addition, $\alpha$-MSH-derived peptides have antipyretic activity through binding to MC4R [21] (Table 1). Finally, by binding to MC4R, melanocortins reduce apoptosis [22], oxidative stress and DNA damage induced by UV radiation in the skin [23] (Table 1). From this, we predict that darker eumelanic individuals should have better anti-inflammatory, antipyretic and anti-oxidative responses than lighter individuals. Only one study has investigated immune response against a specific nonpathogenic antigen and found that darker individuals mount a better antibody response [5] (Table 2).

\section{Melanin-based coloration and energy homeostasis}

Melanocortins (especially $\alpha$ - and $\beta$-MSH as well as their endogenous inverse agonist and antagonist AGRP) play a pivotal role in the central and peripheral control of energy homeostasis. In response to increased insulin and leptin inputs, melanocortins binding to neural MC3R and MC4R reduce food intake and coordinately stimulate energy expenditure by inducing the production of thyroid hormones, enhancing metabolic rate and increasing physical activity (Table 1). AGRP has the opposite effects [24]. Thus, melanocortin activity promotes reduction of adult adipose mass tissue and ultimately prevents adult overweight (Table 1). Melanocortins also enhance energy expenditure through diet-induced thermogenesis [25]. In addition to their regulatory effect on energy intake and expenditure, melanocortins also regulate glucose homeostasis (Table 1). Based on these effects, darker eumelanic individuals should be better able to maintain the energy balance between food intake and energy expenditure. In the two cases where this has been studied, as predicted, darker eumelanic individuals had a higher resting metabolic rate (Table 2).

\section{Other pleiotropic effects of melanocortins}

In addition to the phenotypic traits discussed above, melanocortins affect other traits, but in directions that are inconsistent across studies. Although for some traits the number of genetic and pharmacological studies is clearly insufficient, we nevertheless briefly report the effects of melanocortins to encourage further research in these directions.

Melanocortins affect growth (through binding to MC2$4 \mathrm{R}$ ), sodium excretion (MC3R) and sleep in vertebrates, although we cannot readily predict whether melanocortins reduce or enhance these physiological processes owing to the paucity of experimental studies (Supplementary Table $\mathrm{S} 1$ ). The modulatory effect of melanocortins on the nervous system (MC3R and MC4R), nociception (MC1R and MC4R) and prolactin excretion depends on the model, food supply and steroid levels, as well as the dosage and route of administration of melanocortin agonists and antagonists (Supplementary Table S1). With respect to adult body size and growth hormones, melanocortins induce opposite effects when binding to different MCRs, with knockout mice for $M C 3 R$ being shorter and knockout mice for $M C 4 R$ or $P O M C$ and dominant agouti mutant mice being larger than wild-type mice [26,27] (Supplementary Table $\mathrm{S} 1$ ). It is therefore difficult to predict the sign of covariation, which could be species and condition dependent in wild populations [28]. In seven studies of vertebrates, darker eumelanic individuals were larger whereas in one study they were smaller than lighter-coloured individuals of the same population (Table 2). Finally, melanocortins enhance grooming, stretching and yawning behaviour, increase cardiovascular tone and favour nerve regeneration in mammals through binding to neural MC4R (Table 1).

\section{Evolutionary implications}

As melanocortins bind to five MCRs located in many tissues and involved in a wide range of physiological and behavioural functions, they can account for the widespread association between melanin-based coloration and other phenotypic traits in wild vertebrates (Table 2). On the basis of the available experimental genetic and pharmacological studies (Table 1), we derived predictions on how melanin-based coloration might covary with five categories of traits including sexual behaviour, aggressiveness and exocrine gland activity, HPA stress response, immune function and energy homeostasis. These predictions hold only when variation in melanin-based coloration is mediated by variation in the level of the agonists at MC1R and the levels of melanocortins are coordinated across tissues (Box 1). By contrast, there should be no consistent association between melanin-based coloration and other phenotypic traits when variation in coloration is due to mutations at effectors of melanogenesis such as $M C 1 R$, which is the case in half of the studied species (Supplementary Table S3). In this respect, it is important to note that variation in melanin-based coloration between human populations is primarily due to mutations at, for example, MC1R, TYR, MATP and SLC24A5 [29,30] and that human populations are therefore not expected to consistently exhibit the associations between melaninbased coloration and the physiological and behavioural traits reported in our study.

Although between-individual variation in melanocortin activity might be primarily responsible for covariations between melanin-based coloration and other phenotypic traits, it is possible that other substances implicated in the melanocortin system also play a role, in particular if they bind to several MCRs (e.g. $\beta$-defensin [31]) or if they are derived from the POMC prohormone (e.g. endorphins which, through binding to opioid receptors, induce eumelanin synthesis [32] and modulate stress response [33]). It 


\section{Box 1. Coordination of the melanocortin system across tissues}

Melanocortin-based covariation between melanin coloration and other phenotypic traits relies on the assumption that the level of activity of the MCRs and their agonists are correlated across tissues. For example, if melanocortins are responsible for the covariation between melanin-based coloration and energy expenditure, the activity of melanocortins in the skin should be correlated with the activity of cerebral melanocortins controlling energy balance. There is, unfortunately, little information on such correlations because melanocortins act at specific time windows during hair and feather growth [46], are rapidly degraded in the blood and are inefficient at crossing the blood-brain barrier [13]. However, whereas earlier studies have failed to demonstrate any relationship between circulating melanocortins and skin pigmentation except under pathological conditions [3], recent studies indicate that almost all skin cells produce melanocortins and all five MCRs, and that the skin functions as a neuroendocrine tissue that communicates with the vascular and neural systems [47]. Accordingly, humans and horses exposed to sunlight exhibit increased levels of melanocortins in both the blood and the skin [48]. Thus, the activity of the melanocortin system can be locally regulated and coordinated across organs by neuroendocrine communication [49]. Interestingly, in Arctic charr ( $S$. alpinus), stress due to social subordination results in increased plasma melanocortin concentrations and skin darkening [50]. Hormones, cytokines and neuropeptides such as corticotropin-releasing hormone, glucocorticoids, sexual steroids, vitamin D, serotonin, melatonin, catecholamines and thyroid hormones, which are also produced locally in the skin and which control the production of melanocortins as well as of MCRs, might be the key regulators that coordinate melanocortin activity and MCR levels in different tissues $[3,49,51]$.

will be important to consider the potential role of these substances to identify the genetic mechanisms underlying the association between melanin-based coloration and other physiological and behavioural traits in vertebrates. Because the POMC gene is highly conserved in vertebrates, variation in the level of melanocortins might not result from polymorphism at this gene but probably from variation in the level of regulatory factors responsible for its transcription and posttranslational modifications (Box 1). The vast array of pleiotropic effects of the POMC gene might constrain its evolution in contrast to $M C 1 R$, which has very few known pleiotropic effects. This might explain why POMC evolves less rapidly than $M C 1 R[34,35]$ and why melanin-based colour morphs with primarily a camouflage function are generally mediated by mutations in the coding region of $M C 1 R$ [36].

The finding that regulators of the melanocortin system can pleiotropically affect the expression of suites of correlated phenotypic traits has important implications with regard to the existence of behavioural syndromes. These syndromes are analogous to personality differences with some individuals being bolder or more shy, and with individuals consistently differing with respect to many traits across a range of situations and contexts [37]. Because flexible behaviours might allow individuals to adjust to each prevailing environmental and social situation, the existence of behavioural syndromes was considered a paradox until recently. A new model based on lifehistory tradeoffs has, however, demonstrated that the evolution of behavioural syndromes can result in polymorphic populations with individuals developing alternative strategies to cope with conflicting situations [38]. Our study suggests that pleiotropic effects could underlie behavioural syndromes, with different strategies being signalled by differences in melanin-based coloration. It remains to be studied whether the association between coloration and suites of physiological and behavioural traits is adaptive. For example, it would be interesting to determine whether the higher level of aggressiveness, sexual activity, anti-inflammatory response and resistance to stress of darker eumelanic individuals is adaptive given that it can be useful for bolder individuals to be more explorative and risk taking [38].

The fact that melanin-based coloration is frequently associated with other linked phenotypic traits has important implications for studies of sexual selection. Thus, preferential choice for lighter or darker individuals might reflect preferential choice for coloration and/or any of the other associated traits. To understand the benefit of mate choice thus requires understanding the effect of all the associated traits on fitness as well as to determine the relative role of each of these traits during mate choice. In that respect it is important to note that, although darker eumelanic individuals might be sexually more active,

\section{Box 2. Future studies}

Below we list potential studies to examine the role of the melanocortin system in generating covariance between eumelanin-based coloration and other phenotypic traits.

(i) Identify covariation between the degree of eumelanin-based coloration and traits including sexual activity, aggressiveness, exocrine gland activity, stress responses, immune function and energy homeostasis. For example: are darker eumelanic individuals more resistant to parasites than lightly coloured conspecifics?

(ii) Identify age-related covariation between eumelanin-based coloration and other phenotypic traits. For example: do individuals that become darker with age also become more immunocompetent?

(iii) Manipulate environmental factors to determine the reaction norm of differently coloured individuals. For example: is growth rate in more and less stressful environments influenced by the degree of coloration?

(iv) Measure melanocortin levels in several tissues and environmental situations in relation to eumelanin-based coloration. For example: is the level of melanocortins involved in melanin production correlated with the level of melanocortins involved in immunity in other organs or tissues?

(v) Determine whether the level of melanocortins is condition dependent. For example: do melanocortin levels vary in relation to experimental manipulation of ecological or social factors?

(vi) Manipulate the level of melanocortins to determine whether these hormones simultaneously affect eumelanin-based coloration and other traits known to covary with eumelaninbased coloration. For example: does injection of melanocortins affect coloration and anti-inflammatory response simultaneously?

(vii) Determine whether variation in the degree of eumelaninbased coloration is associated with genetic variation of genes involved in melanin synthesis. For example: is interindividual variation in eumelanin-based coloration associated with mutations at MC1R or POMC?

(viii) Identify the genetic mechanisms leading to variation in the production of melanocortins. For example: is interindividual variation in melanocortin levels due to a polymorphism at the $P O M C$ gene, to variation in the expression level of the POMC gene or to posttranslational modification of the POMC prohormone? 
aggressive and resistant to stressful environments, it does not necessarily imply that they are superior to lighter conspecifics, as some of these phenotypic attributes might also entail substantial costs $[39,40]$. Moreover, the fitness consequences of choosing a partner expressing some of these phenotypic traits might vary over space and time, possibly explaining why partner choice is sometimes context dependent [41,42]. A challenge will be to identify how ecological, social and physiological factors jointly influence and maintain interindividual variation in melanin-based coloration and associated phenotypic traits. This endeavour will require a combination of pharmacological, medical, ecological and evolutionary approaches using model laboratory organisms and wild populations of vertebrates (Box 2).

Finally, we would like to stress that the framework presented here is not limited to the melanin synthesis pathway. Many hormones and peptides have promiscuous properties making them likely candidates for mediating, via pleiotropic effects, covariations between important phenotypic and behavioural traits. For example, the neuropeptide Y (NPY), which is the most abundant peptide in the mammalian brain, binds to at least five $G$ protein receptors (Y1, Y2, Y4, Y5 and Y6) which control very different biochemical pathways. Accordingly, NPY has potent effects on traits as diverse as feeding, body weight, blood pressure, innate immunity function and behavioural domains such as motor activity and anxiety [43,44]. It would be of great interest to employ the methodology used in the present study to determine whether these traits covary in a manner consistent with the known functions of the five $\mathrm{Y}$ receptors and between-individual variation in NPY levels. More generally, we hope that our study will elicit similar investigations of other hormones and peptides with promiscuous properties with the aim of clarifying whether pleiotropic effects are an important cause underlying suites of correlated traits (e.g. [45]) and pinpointing other covariations between important phenotypic and behavioural phenotypes.

\section{Acknowledgements}

We thank the Swiss National Science Foundation for financial support (to A.R. and L.K.), Alex N. Eberle, Nick Mundy, Karen Parker, Thierry Pedrazzini, François Pralong, Marc Robinson-Rechavi, Andrzej Slominski, Bernard Thorens, Jean-Nicolas Volff and four anonymous reviewers for helpful comments on a previous version of the manuscript.

\section{Supplementary data}

Supplementary data associated with this article can be found, in the online version, at doi:10.1016/j.tree.2008. 06.001 .

\section{References}

1 Majerus, M.E.N. (1998) Melanism: Evolution in Action, Oxford University Press

2 Roulin, A. (2004) The evolution, maintenance and adaptive function of genetic colour polymorphism in birds. Biol. Rev. 79, 815-848

3 Slominski, A. et al. (2004) Melanin pigmentation in mammalian skin and its hormonal regulation. Physiol. Rev. 84, 1155-1228

4 West, P.M. and Packer, C. (2002) Sexual selection, temperature, and the lion's mane. Science 297, 1339-1343

5 Roulin, A. et al. (2000) Female barn owls (Tyto alba) advertise good genes. Proc. R. Soc. Lond. B Biol. Sci. 267, 937-941
6 Schioth, H.B. et al. (2005) Evolutionary conservation of the structural, pharmacological, and genomic characteristics of the melanocortin receptor subtypes. Peptides 26, 1886-1900

7 Pritchard, L.E. and White, A. (2007) Neuropeptide processing and its impact on melanocortin pathways. Endocrinology 148, 4201-4207

8 Kingo, K. et al. (2007) Gene expression analysis of melanocortin system in vitiligo. J. Dermatol. Sci. 48, 113-122

9 Lin, J.Y. and Fisher, D.E. (2007) Melanocyte biology and skin pigmentation. Nature 445, 843-850

10 Dinulescu, D.M. and Cone, R.D. (2000) Agouti and agouti-related protein: analogies and contrasts. J. Biol. Chem. 275, 6695-6698

11 Stutz, A.M. et al. (2005) The Agouti-related protein and its role in energy homeostasis. Peptides 26, 1771-1781

12 Shadiack, A.M. et al. (2007) Melanocortins in the treatment of male and female sexual dysfunction. Curr. Top. Med. Chem. 7, 1137-1144

13 Eberle, A.N. (1988) The Melanotropins: Chemistry, Physiology and Mechanism of Action, Karger

14 Morgan, C. et al. (2004) Melanocortin-5 receptor deficiency reduces a pheromonal signal for aggression in male mice. Chem. Senses 29, 111115

15 Chen, W. et al. (1997) Exocrine gland dysfunction in MC5-R-deficient mice: evidence for coordinated regulation of exocrine gland function by melanocortin peptides. Cell 91, 789-798

16 Roulin, A. (2007) Melanin pigmentation negatively correlates with plumage preening effort in barn owls. Funct. Ecol. 21, 264-271

17 Charmandari, E. et al. (2005) Endocrinology of the stress response. Annu. Rev. Physiol. 67, 259-284

18 Slominski, A. et al. (2007) Differential expression of HPA axis homolog in the skin. Mol. Cell. Endocrinol. 265, 143-149

19 Racca, S. et al. (2005) Effects of swim stress and $\alpha$-MSH acute pretreatment on brain 5-HT transporter and corticosterone receptor. Pharmacol. Biochem. Behav. 81, 894-900

20 Getting, S.J. (2006) Targeting melanocortin receptors as potential novel therapeutics. Pharmacol. Ther. 111, 1-15

21 Roth, J. et al. (2004) Endogenous antipyretics: neuropeptides and glucocorticoids. Front. Biosci. 9, 816-826

22 Chai, B. et al. (2006) Melanocortin-4 receptor-mediated inhibition of apoptosis in immortalized hypothalamic neurons via mitogenactivated protein kinase. Peptides 27, 2846-2857

23 Bohm, M. et al. (2005) $\alpha$-Melanocyte-stimulating hormone protects from ultraviolet radiation-induced apoptosis and DNA damage. J. Biol. Chem. 280, 5795-5802

24 Cone, R.D. (2006) Studies on the physiological functions of the melanocortin system. Endocr. Rev. 27, 736-749

25 Fan, W. et al. (2005) Regulation of thermogenesis by the central melanocortin system. Peptides 26, 1800-1813

26 Butler, A.A. and Cone, R.D. (2002) The melanocortin receptors: lessons from knockout models. Neuropeptides $36,77-84$

27 Yen, T.T. et al. (1994) Obesity, diabetes, and neoplasia in yellow $A^{(v y)} /-$ mice: ectopic expression of the agouti gene. FASEB J. 8, 479-488

28 Roulin, A. et al. (2008) Melanin-based colorations signal strategies to cope with poor and rich environments. Behav. Ecol. Soc. 62, 507-519

29 Lao, O. et al. (2007) Signatures of positive selection in genes associated with human skin pigmentation as revealed from analyses of single nucleotide polymorphisms. Ann. Hum. Genet. 71, 354-369

30 Sturm, R.A. (2006) A golden age of human pigmentation genetics. Trends Genet. 22, 464-468

31 Candille, S.I. et al. (2007) A $\beta$-defensin mutation causes black coat color in domestic dogs. Science 318, 1418-1423

32 Kauser, S. et al. (2004) $\beta$-Endorphin as a regulator of human hair follicle melanocyte biology. J. Invest. Dermatol. 123, 184-195

33 Vaanholt, L.M. et al. (2003) $\beta$-Endorphin modulates the acute response to a social conflict in male mice but does not play a role in stressinduced changes in sleep.. Brain Res. 978, 169-176

$34 \mathrm{Li}$, X.L. et al. (2007) Evolution and differentiation of MSHR gene in different species. J. Hered. 98, 165-168

35 Dores, R.M. and Lecaude, S. (2005) Trends in the evolution of the proopiomelanocortin gene. Gen. Comp. Endocrinol. 142, 81-93

36 Hoekstra, H.E. et al. (2006) A single amino acid mutation contributes to adaptive beach mouse color pattern. Science 313, 101-104

37 Sih, A. et al. (2004) Behavioral syndromes: an ecological and evolutionary overview. Trends Ecol. Evol. 19, 372-378 
38 Wolf, M. et al. (2007) Life-history trade-offs favour the evolution of animal personalities. Nature 447, 581-584

39 Bonneaud, C. et al. (2003) Assessing the cost of mounting an immune response. Am. Nat. 161, 367-379

40 Krakauer, D.C. and Nowak, M. (1999) T-cell induced pathogenesis in HIV: bystander effects and latent infection. Proc. Biol. Sci. 266, 10691075

41 Jawor, J.M. and Breitwisch, R. (2003) Melanin ornaments, honesty, and sexual selection. Auk 120, 249-265

42 Roulin, A. and Bize, P. (2007) Sexual selection in genetic colourpolymorphic species: a review of experimental studies and perspectives. J. Ethol. 25, 99-105

43 Pedrazzini, T. et al. (2003) Neuropeptide Y: the universal soldier. Cell. Mol. Life Sci. 60, 350-377

44 Wheway, J. et al. (2007) NPY and receptors in immune and inflammatory diseases. Curr. Top. Med. Chem. 7, 1743-1752

45 Blows, M.W. and Brooks, R. (2003) Measuring nonlinear selection. Am. Nat. $162,815-820$
46 Slominski, A. et al. (2000) Corticotropin releasing hormone and proopiomelanocortin involvement in the cutaneous response to stress. Physiol. Rev. 80, 979-1020

47 Slominski, A. (2005) Neuroendocrine system of the skin. Dermatology 211, 199-208

48 Zbytek, B. et al. (2006) Characterization of a ultraviolet B-induced corticotropin-releasing hormone-proopiomelanocortin system in human melanocytes. Mol. Endocrinol. 20, 2539-2547

49 Slominski, A. and Wortsman, J. (2000) Neuroendocrinology of the skin. Endocr. Rev. 21, 457-487

50 Hoglund, E. et al. (2000) Skin darkening, a potential social signal in subordinate Arctic charr (Salvelinus alpinus): the regulatory role of brain monoamines and pro-opiomelanocortin-derived peptides. J. Exp. Biol. 203, 1711-1721

51 Rousseau, K. et al. (2007) Proopiomelanocortin (POMC), the ACTH/ melanocortin precursor, is secreted by human epidermal keratinocytes and melanocytes and stimulates melanogenesis. FASEB J. 21, 1844 1856 